\title{
Aerodynamic Analysis on Postures of Ski Jumpers during Flight using Computational Fluid Dynamics*
}

\author{
Minhyoung RYU, ${ }^{1)}$ Leesang $\mathrm{CHO},{ }^{2)}$ and Jinsoo $\mathrm{CHO}^{3)}$ \\ ${ }^{1)}$ Department of Mechanical Engineering, Hanyang University, Seoul 133-791, Korea \\ ${ }^{2)}$ Department of Mechanical Systems Engineering, Hansung University, Seoul 136-792, Korea \\ ${ }^{3}$ School of Mechanical Engineering, Hanyang University, Seoul 133-791, Korea
}

\begin{abstract}
The aerodynamic characteristics of a ski jumper model in various postures are analyzed using a commercial computational fluid dynamics tool. The purpose of this study is to understand the aerodynamic characteristics of ski jumping. The range of flying postures for ski jumpers is determined as widely as possible to simulate the optimal flight position of ski jumpers. The computational results are in good agreement with experimental results. The aerodynamic characteristics are influenced by the hip and body angles of the ski jumper model during the flight. The lift-to-drag ratio of ski jumpers is increased as hip angle increases. However, as the hip angle increases, the region of the angle of attack becomes restricted for stable flight. The augmentation of the body angle can enhance the most favorable angle of attack, because the body angle is likely to result in a cambered airfoil. In conclusion, the ski jumper should maintain a high hip angle with an angle of attack that stabilizes the flight condition, to improve flight distance.
\end{abstract}

Key Words: Ski Jumping, CFD Analysis, Lift-to-drag Ratio

\section{Nomenclature}

$A_{p}:$ cross-sectional area

$C_{L}$ : lift coefficient

$C_{D}$ : drag coefficient

$F_{D}$ : drag force

$F_{L}$ : lift force

$h_{\text {tot }}$ : specific total enthalpy

$l$ : ski length

$L / D$ : lift-to-drag ratio

$L / D_{\max }$ : maximum lift-to-drag ratio

$P$ : static pressure

$S_{E}$ : energy source

$S_{M}$ : momentum source

$t$ : time

$U$ : mean velocity vector

$v$ : free stream velocity

$V$ : ski angle

$y+$ : dimensionless wall distance

$\alpha$ : angle of attack

$\beta$ : body angle relative to the skis

$\gamma$ : hip angle

$\lambda$ : thermal viscosity

$\mu$ : molecular (dynamic) viscosity

$\rho$ : air density

$\tau$ : mean viscous stress tensor

$\varphi$ : arm angle

\section{Introduction}

The 23rd Winter Olympic Games will be held at Pyeongchang-Gun in the Republic of Korea in 2018. In the Republic of Korea, many people are interested in ski jumping because it is one of the representative sports of the Winter Olympic Games.

Ski jumping is affected by the aerodynamic characteristics of the flying postures. Ski jumpers go down the slope and take off at the springboard. The style of the ski jumper is evaluated by judges and the distance is measured by referees. Commonly, the flight distance is more important more than the flying style. ${ }^{1)}$ To increase the length of a jump, the liftto-drag ratio should be increased.

In the beginning of the 20th century, systematic research on ski jumping was started in developed countries where winter sports are popular, such as Switzerland, Austria and Japan.

Straumann ${ }^{2)}$ initiated the study of ski jumping in 1926 . He conducted studies to determine the most advantageous flying posture using wind tunnel tests and the calculation of the flight path.

The first study of a systematic simulation jump was performed by Hochmuth ${ }^{3)}$ in 1958. He investigated the biomechanical properties for flight trajectories using cinematographic techniques. Various simulation studies of ski jumping using computational methods have been actively performed along with wind tunnel tests.

A variety of computer simulations ${ }^{4-6)}$ provide the configuration information of the hill and the optimized flight attitude of the jumping athletes for long-distance jumps. They presented the optimized flight attitude of jumping athletes. The computational simulation study of the flight trajectory of ski jumping led to the construction of an aerodynamics database of field studies and wind tunnel tests.
(C) 2015 The Japan Society for Aeronautical and Space Sciences *Received 27 March 2014; final revision received 19 September 2014; accepted for publication 15 January 2015. 
In theoretical studies, Remizov ${ }^{7)}$ and Denoth et al. ${ }^{8)}$ investigated how to maximize the flight distance using computational simulations based on wind tunnel test results.

Müller et al. ${ }^{9)}$ measured the aerodynamic force of a worldclass ski jumper in various flight positions using wind tunnel testing. They deduced highly reliable mapping results from field tests performed during the World Championships in the Ski Flying 1994 at Planica (Slovenia).

Schmölzer and Müller ${ }^{10)}$ performed field tests during the 1999/2000 World Cup Games at Villach (Austria). They performed wind tunnel tests with 1:1 scale models of the athletes to compare with computer simulation results for the flight phase. The body weight of the ski jumper, the air density, and the wind gust were considered for analysis in the flight phase. In their simulation results, the flight distance of the ski jumper is increased with the reduction of the body weight along with an increase in both the air density and the flight altitude.

Schmölzer and Müller ${ }^{11)}$ secured a database for the field test results of elite ski jumpers during the 2002 Winter Olympic Games at Salt Lake City (USA). The individual flight styles of the ski jumpers were analyzed using 11 pieces of camera equipment. The computer simulation evaluated the influence of the body mass index of the athletes on the flight distance and the flight style of the athletes at high altitude $(2,000 \mathrm{~m})$. The results of their study indicated that the flight distance is longer when the hip angle $(\gamma)$ of the ski jumper is less than $160^{\circ}$ and the ski angle $(V)$ is below $35^{\circ}$.

Meile et al. ${ }^{12)}$ analyzed the aerodynamic characteristics of ski jumping postures and ski suits using wind tunnel tests and CFD analysis. The basis for changes in regulations was inferred with respect to safety and health improvements. In addition, commercial CFD tools were assessed regarding expectations of the aerodynamic characteristics for a ski jumper during flight.

Jin et al. ${ }^{13)}$ performed a study regarding the V-style flight, which is a new flight style developed by a Swedish ski jumper in 1989. They studied the parallel style, the V-style and the flat $\mathrm{V}$-style for the flight postures. The most effective flight style is the flat $\mathrm{V}$-style.

Watanabe and Watanabe ${ }^{14)}$ conducted a wind tunnel test to measure the torque and the aerodynamic forces of the 1:1 scale model for the V-style flight. They confirmed that the $\mathrm{V}$-style flight posture positively influences the jumping flight distance.

Seo et al. ${ }^{15)}$ performed a wind tunnel test for the full-size model to analyze the aerodynamic characteristics of the ski jumper model during flight under aerodynamic stall. In the case of a high angle of attack, the lift force is highly increased during flight using the $\mathrm{V}$-style compared with that of using the parallel style. The V-style flight of the ski jumper results in increased lift and reduced drag compared with those of the other styles during flight.

Seo et al. ${ }^{16)}$ performed a numerical analysis to determine the optimal flight postures in the V-style. The database of the aerodynamic characteristics for the ski jumper model ac- cumulated during wind tunnel testing was used to simulate the flight trajectory.

Marqué-Bruna and Grimshaw ${ }^{17)}$ analyzed flight postures to understand the mechanisms of flight stability. They proposed improved postures with the ski angle $(V)$ of approximately 30 degrees and a body angle relative to the ski plate $(\beta)$ of approximately 10 degrees.

Most previous ski jumping studies, focus on the optimization of flight postures to enable longer flight distances by using wind tunnel tests and computer simulations. However, optimal flight postures are different for each ski jumper. It is also difficult to determine the best posture for the ski jumpers during flight.

In the present study, we focus on a better understanding of the aerodynamic characteristics for ski jumping during flight. The aerodynamic characteristics of the ski jumper model for various flight postures are analyzed using commercial CFD software. Validation of the ski jumper model during the flight was performed by comparing actual results with the experimental results. ${ }^{11)}$

\section{Computational Method}

\subsection{Ski jumper model}

Typical flight postures for the CFD simulation of the ski jumper model were selected based on the results of Schmölzer and Müller ${ }^{10,11)}$ and Meile et al. ${ }^{12)}$

The angle of attack $(\alpha)$, body angle $(\beta)$ relative to the ski plate, hip angle $(\gamma)$, ski angle $(V)$ and arm angle $(\varphi)$ for the ski jumper model are measured relative to the airstream as shown in Fig. 1.

A three-dimensional ski jumper model is considered based on the typical body shapes of Korean adult males. The dimensions of the ski jumper model and the ski equipment are listed in Table 1. The size of the ski equipment is determined by FIS regulations. ${ }^{18)}$

Previous research ${ }^{12)}$ indicated that world-class ski jumpers have a hip angle between $140^{\circ}$ and $160^{\circ}$. In this paper, the

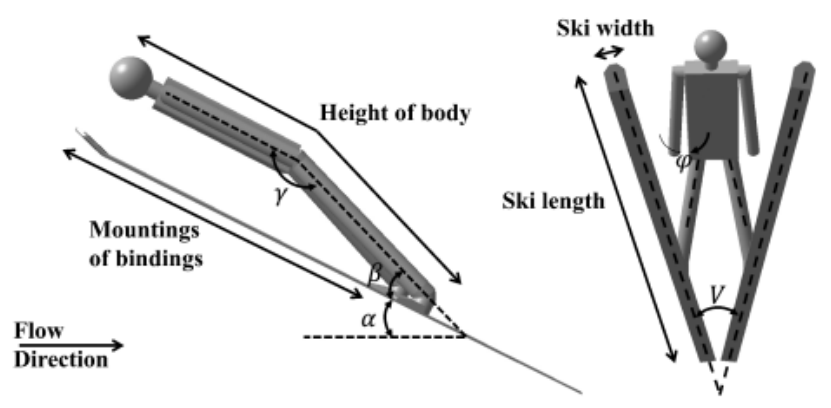

Fig. 1. Typical posture and flight position for the CFD simulation of the ski jumper model.

Table 1. Dimensions of the ski jumper model for a Korean national team athlete and the ski equipment.

\begin{tabular}{cccc}
\hline $\begin{array}{c}\text { Height of } \\
\text { body }\end{array}$ & Ski length & $\begin{array}{c}\text { Mounting of } \\
\text { binding }\end{array}$ & Ski width \\
\hline $1.700 \mathrm{~m}$ & $2.470 \mathrm{~m}$ & $1.410 \mathrm{~m}$ & $0.115 \mathrm{~m}$ \\
\hline
\end{tabular}


Table 2. The range of position parameters $(\alpha, \beta, \gamma, V)$.

\begin{tabular}{lccccc}
\hline Parameter & $\alpha(\mathrm{deg})$ & $\beta(\mathrm{deg})$ & $\gamma(\mathrm{deg})$ & $V(\mathrm{deg})$ & $\varphi(\mathrm{deg})$ \\
\hline Range & $0-45$ & $10-30$ & $140-160$ & $0-35$ & $2.5-10$ \\
Increments & 5 & 5 & 10 & - & \\
\hline
\end{tabular}

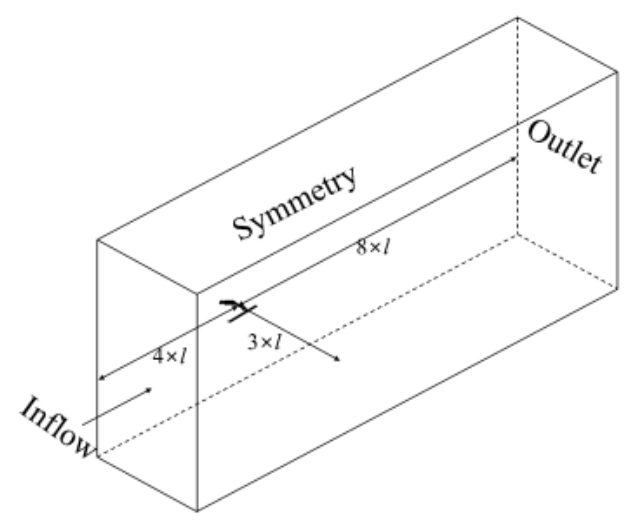

Fig. 2. Computational domain and boundary conditions.

hip angle $(\gamma)$ range of the ski jumper model is from $140^{\circ}$ to $160^{\circ}$.

The range of position parameters used in this study are presented in Table 2.

\subsection{Computational domain and boundary conditions}

Figure 2 shows the computational domain and boundary conditions for CFD simulation of the ski jumper model. The computational domain for the ski jumper model is set as the front region of length $4 \times l$, rear region of length $8 \times l$ and left region of length $3 \times l$, where, the ski length $(l)$ is $2.470 \mathrm{~m}$.

The symmetry condition of the computational domain is used to reduce computational time. To account for the wake effect, the ski jumper model is located at a position of about $33 \%$ of the computational domain. Crosswind effects are neglected because this velocity is smaller than the free-stream velocity.

The range of flight velocity is commonly from 25 to $30 \mathrm{~m} / \mathrm{s}$ for ski jumping, based on the height of the jumping and flight phase. $^{12)}$ The free-stream velocity is set to $25 \mathrm{~m} / \mathrm{s}$. The surface of the ski jumper model is applied using the no-slip condition and the outside areas are set to free-slip boundary conditions.

\subsection{Governing equations and turbulence model}

The aerodynamic characteristics for the ski jumper model were analyzed using commercial software (ANSYS CFX Ver. 14.5). The CFD simulation was performed for the steady-state flow. To predict the three-dimensional viscous turbulent flows and aerodynamic characteristics of the ski jumper model, the governing equations (continuity, momentum and energy equations) are expressed as the Reynolds Averaged Navier-Stokes (RANS) equations as follows. ${ }^{19)}$ Continuity equation:

$$
\frac{\partial \rho}{\partial t}+\nabla \cdot(\rho U)=0
$$

Momentum equation:

$$
\frac{\partial(\rho U)}{\partial t}+\nabla \cdot(\rho U \times U)=-\nabla p+\nabla \cdot \tau+S_{M}
$$

Energy equation:

$$
\begin{aligned}
& \frac{\partial\left(\rho h_{t o t}\right)}{\partial t}-\frac{\partial p}{\partial t}+\nabla \cdot\left(\rho U h_{t o t}\right) \\
& \quad=\nabla \cdot(\lambda \nabla T)+\nabla \cdot(U \cdot \tau)+U \cdot S_{M}+S_{E}
\end{aligned}
$$

The pressure-based method with the $\kappa-\omega S S T$ turbulence model $^{20)}$ was used in this study. The $\kappa-\omega S S T$ turbulence model is well known for its ability to predict the adverse pressure gradient, flow separation near the wall and shear flow stress. ${ }^{21-23)}$ This turbulence model makes use of the $\kappa-\varepsilon$ turbulence model ${ }^{24)}$ and $\kappa-\omega$ turbulence model. ${ }^{25)}$ The $\kappa-\omega$ turbulence model was used in the sub- and log-layer. The standard $\kappa-\varepsilon$ model was applied in the wake region of the boundary layer. Furthermore, the eddy viscosity was determined using a modified version of the Johnson-King model. ${ }^{26)}$

The lift-to-drag ratio is an important conventional parameter in the aerodynamic performances of the ski jumper model. By analyzing the pitching moment coefficient, the static longitudinal stability of the ski jumper model is evaluated under flight conditions. The relevant aerodynamic coefficients are expressed as follows.

Lift coefficient:

$$
C_{L}=\frac{F_{L}}{1 / 2 \rho v^{2} A_{P}}
$$

Drag coefficient:

$$
C_{D}=\frac{F_{D}}{1 / 2 \rho v^{2} A_{P}}
$$

Lift-to-drag ratio:

$$
L / D=F_{L} / F_{D}
$$

Pitching moment coefficient:

$$
C_{M}=\frac{M}{1 / 2 \rho v^{2} A_{P} l}
$$

\subsection{Grid dependency study}

The computational meshes for the ski jumper model are shown in Fig. 3. In this figure, the computational meshes have $4.2 \times 10^{6}$ elements and the number of nodes is $1.8 \times 10^{6}$. The dimensionless wall distance is less than 1 to consider the viscous sub-layer in the typical velocity profiles for the turbulent boundary layer based on the law of the wall. ${ }^{27)}$ The dimensionless wall distance is calculated when the Reynolds number is $2.56 \times 10^{5}$ using the thickness of the body as the characteristic length. ${ }^{28)}$ The smallest grid size is $1 \mathrm{~mm}$ near the surface of the model. The growth rate of the grid is set at 1.2 with 30 prism layers. The maximum grid size is restricted to $500 \mathrm{~mm}$ at the outer region.

Figure 4 shows the aerodynamic coefficients of the ski jumper model for the grid convergence test. If the number of grids is greater than $4.0 \times 10^{6}$. then the difference of the 


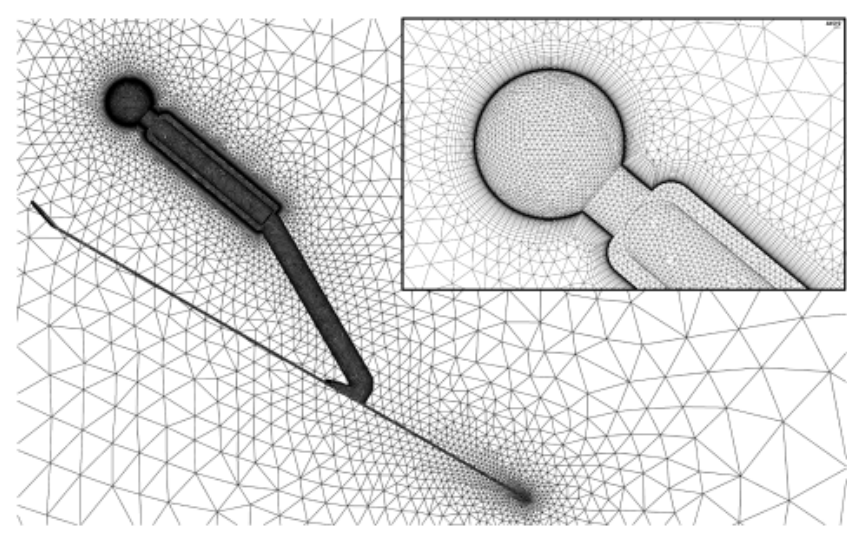

Fig. 3. Hybrid meshes and inflation grids on the boundary layer (number of elements $=4.2 \times 10^{6}$ ).

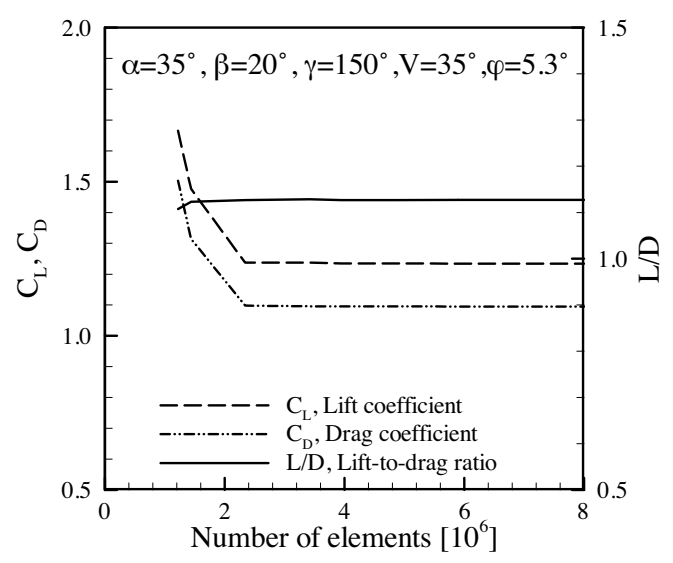

Fig. 4. Grid convergence test results.

lift-to-drag ratio for the ski jumper model is less than $2 \%$. Therefore, the number of grids is determined as $4.2 \times 10^{6}$ to reduce the computing time.

\section{Computational Results}

\subsection{Computational model validation}

In Fig. 5, the lift-to-drag ratio for different flight position angles is plotted and compared with the experimental results measured by Schmölzer and Müller. ${ }^{11)}$

The maximum difference is approximately $7.170 \%$ at $\alpha=35^{\circ}$. The computational results are in good agreement with the experimental results. The difference between the wind tunnel tests and the computational analysis conditions are presented in Table 3. A positioning apparatus was used in the wind tunnel test by Schmölzer and Müller. ${ }^{11)}$ In this study, the area of the computational domain is large enough to eliminate the wall effect of walls while minimizing the blockage effect.

\subsection{Aerodynamic characteristics for various postures}

The lift-to-drag ratio was evaluated for the quality of the lifting components in the flight. Figure 6 shows the results of the lift-to-drag ratio for different flight positions of ski jumping athletes.

The lift-to-drag ratio for the ski jumper model is more influenced by the hip angle than by the other angles. The lift-

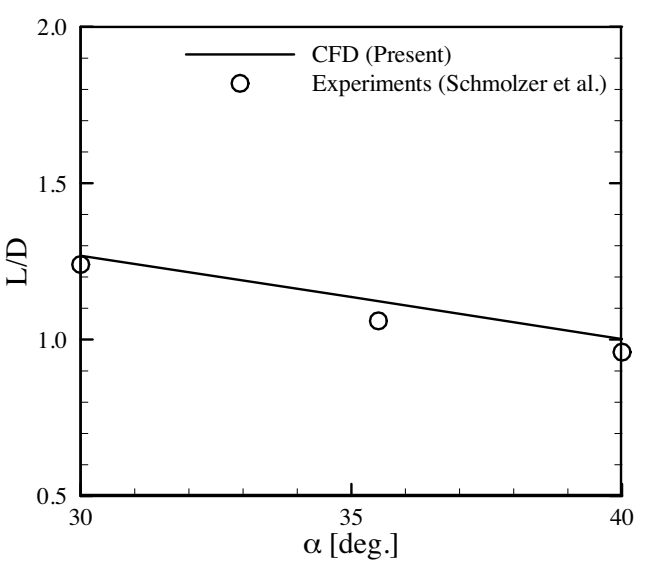

Fig. 5. Comparison of the lift-to-drag ratio between present and Schmölzer and Müller $\left.\left(\beta=15^{\circ}, \gamma=160^{\circ}, V=35^{\circ}, v=5.3^{\circ}\right) .^{11}\right)$

Table 3. Setup environment for the CFD analysis and wind tunnel test.

\begin{tabular}{lcc}
\hline Setup conditions & Present & Schmölzer and Müller ${ }^{11)}$ \\
\hline Model size & $1.70 \mathrm{~m}$ & $1.78 \mathrm{~m}$ \\
Ski length & $2.47 \mathrm{~m}$ & $2.60 \mathrm{~m}$ \\
Reynolds number & $2.56 \times 10^{5}$ & $3.10 \times 10^{5}$ \\
Existence of strut & None & Lower struts \\
\hline
\end{tabular}

Table 4. The maximum lift-to-drag ratio for different hip angles.

\begin{tabular}{cccc}
\hline Postures & $L / D$ & $C_{L}$ & $C_{D}$ \\
\hline$\alpha=15^{\circ}, \beta=20^{\circ}, \gamma=160^{\circ}$, & 1.457 & 0.679 & 0.466 \\
$V=35^{\circ}, \varphi=5.3^{\circ}$ & & & \\
$\alpha=20^{\circ}, \beta=20^{\circ}, \gamma=150^{\circ}$, & 1.379 & 0.804 & 0.583 \\
$\quad V=35^{\circ}, \varphi=5.3^{\circ}$ & & & \\
$\alpha=20^{\circ}, \beta=30^{\circ}, \gamma=140^{\circ}$, & 1.294 & 0.752 & 0.581 \\
$V=35^{\circ}, \varphi=5.3^{\circ}$ & & & \\
\hline
\end{tabular}

to-drag ratio of the ski jumper model is increased by increasing the hip angle. The overall maximum lift-to-drag ratio is 1.457 at $\alpha=15^{\circ}, \beta=20^{\circ}, \gamma=160^{\circ}, V=35^{\circ}$ and $\varphi=5.3^{\circ}$ as presented in Table 4 . This posture is in good agreement with the experimental result by Meile et al. ${ }^{29)}$

The local maximum lift-to-drag ratios are 1.379 and 1.294 at $\gamma=150^{\circ}$ and $\gamma=140^{\circ}$, respectively. At a low angle of attack $\left(\alpha \leq 30^{\circ}: \gamma=140^{\circ}, \alpha \leq 25^{\circ}: \gamma=150^{\circ}, \alpha \leq 20^{\circ}\right.$ : $\left.\gamma=160^{\circ}\right)$, the lift-to-drag ratio is increased by the influence of the body angle. The slope of the lift-to-drag ratio is enhanced by increasing the body angle at a low angle of attack. At the other region, the slope of lift-to-drag ratio rapidly declines. The lift-to-drag ratio is significantly influenced by the body angle in the range of low angle of attack. The effect of the hip angle is significant at a high angle of attack. The effective body angle is located at $\alpha \approx 10-20^{\circ}$. The body angle is similar to the camber of an airfoil; therefore, augmentation of the body angle can enhance both the effective $\alpha$ and the lift-to-drag ratio. The effect of increasing the camber increases the lift-to-drag ratio by a constant increment for each angle of attack in the linear range. ${ }^{30)}$

Figure 7 shows the aerodynamic coefficients of a ski jumper during flight. As shown in Figs. 7(b) and (c), the lift 


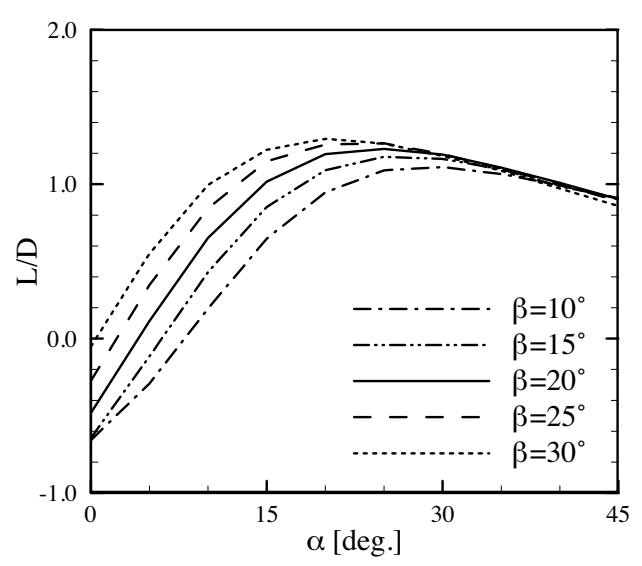

(a) $\gamma=140^{\circ}, V=35^{\circ}, \varphi=5.3^{\circ}$

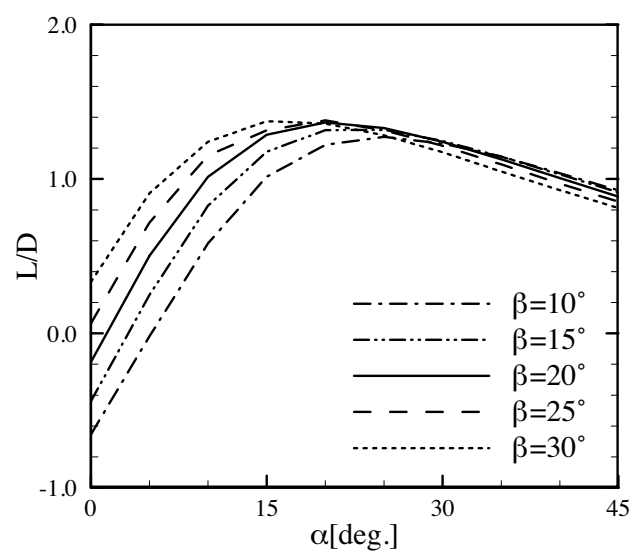

(b) $\gamma=150^{\circ}, V=35^{\circ}, \varphi=5.3^{\circ}$

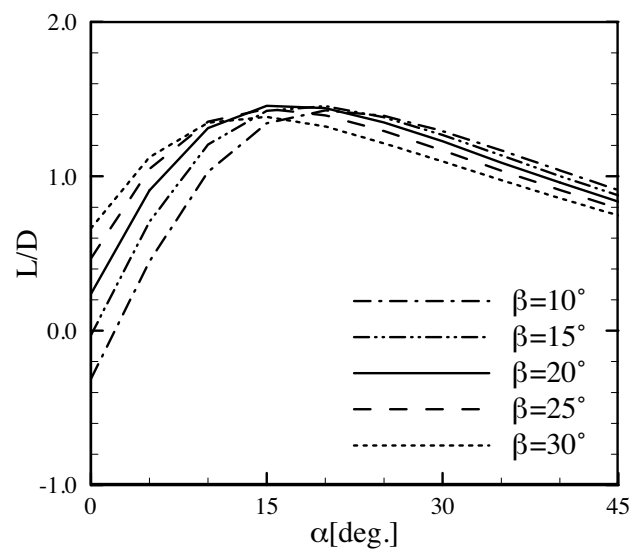

(c) $\gamma=160^{\circ}, V=35^{\circ}, \varphi=5.3^{\circ}$

Fig. 6. Lift-to-drag ratio for various flight postures.

coefficient increased linearly for angle of attack $(\alpha)$ values up to $35^{\circ}$. The lift coefficient is not increased at $\alpha \approx 40^{\circ}$ because aerodynamic stall occurs. The zero-lift drag coefficient is approximately 0.3 during the flight. The drag coefficient decreases as hip angle $(\gamma)$ increases. Each drag polar curve is similar to $C_{D} \leq 1.2$. The drag coefficient significantly increases as hip angle $(\gamma)$ decreases in the range of $C_{D} \geq 1.2$. The increase in body angle $(\beta)$ and hip angle $(\gamma)$ increases the lift coefficient. This increase is similar to the effect of the camber in an airfoil. The $L / D_{\max }$ point means optimal posture for the ski jumper during flight. The optimal point is tangent to the drag polar curve. The maximum lift-to-drag ratio
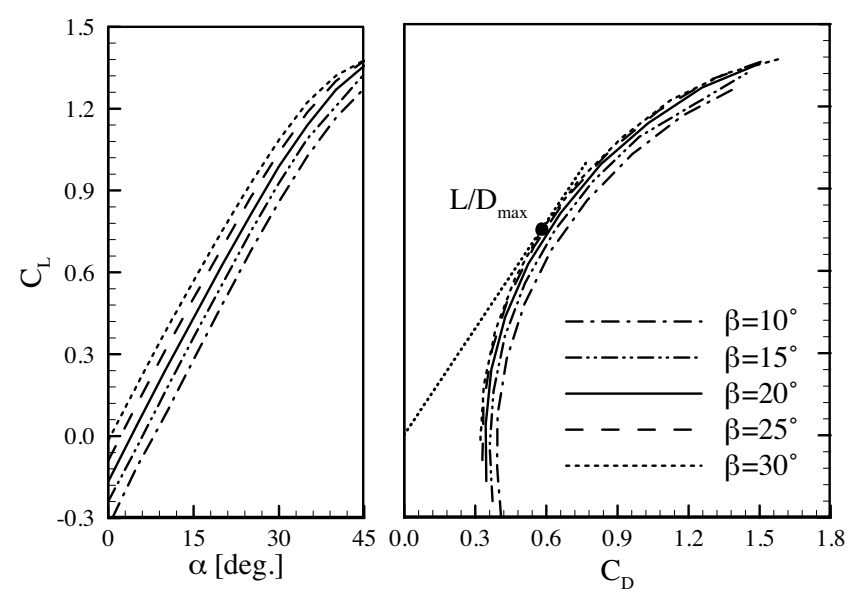

(a) $\gamma=140^{\circ}, V=35^{\circ}, \varphi=5.3^{\circ}$
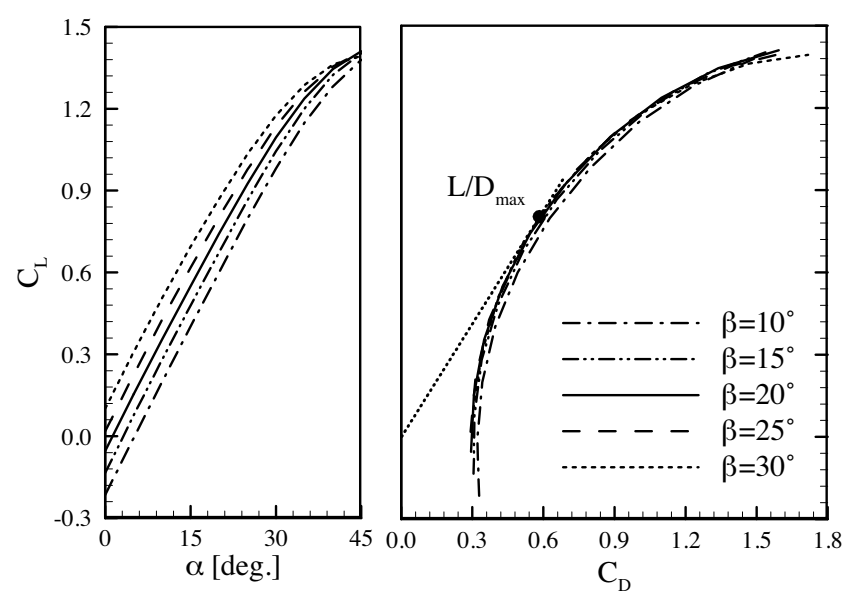

(b) $\gamma=150^{\circ}, V=35^{\circ}, \varphi=5.3^{\circ}$
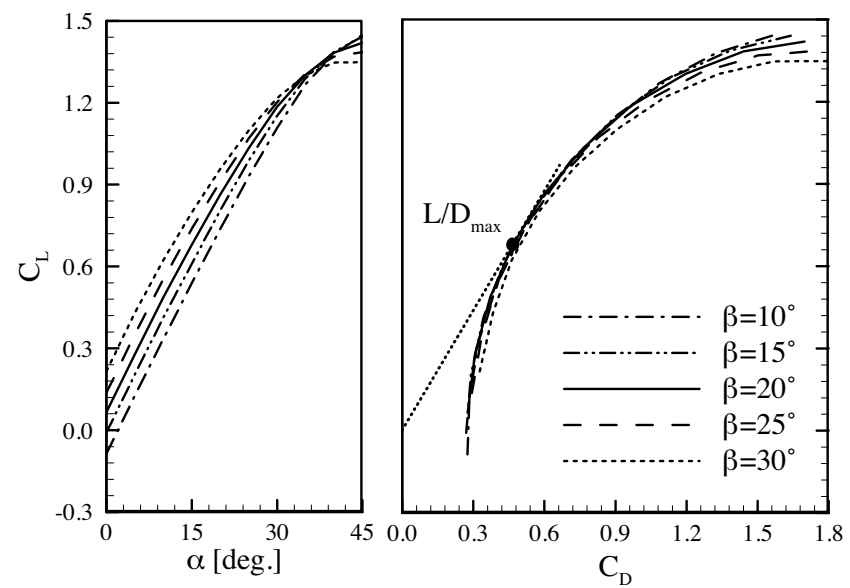

(c) $\gamma=160^{\circ}, V=35^{\circ}, \varphi=5.3^{\circ}$

Fig. 7. Lift and drag coefficients for various flight postures.

point is confirmed for the drag polar curve in Fig. 7. When the hip angle $(\gamma)$ is $160^{\circ}$, the $L / D_{\max }$ is 1.457 . This result is $12.55 \%$ higher than the result of $\gamma=140^{\circ}$.

As shown in Fig. 8, the pitching moment coefficient of the ski jumper model is analyzed for various postures to evaluate the longitudinal stability of the ski jumper model. The pitching moment coefficient is calculated from the center of mass of the ski jumper model. The value of $\mathrm{d} C_{M} / \mathrm{d} \alpha$ must be a negative value to maintain the longitudinal balance and static 


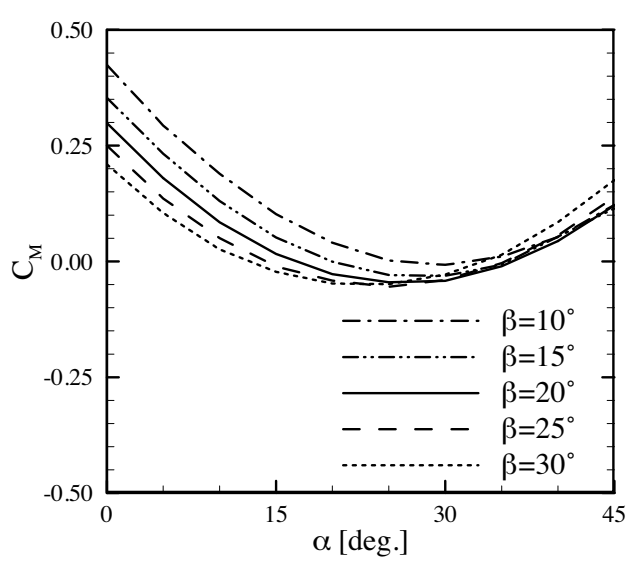

(a) $\gamma=140^{\circ}, V=35^{\circ}, \varphi=5.3^{\circ}$

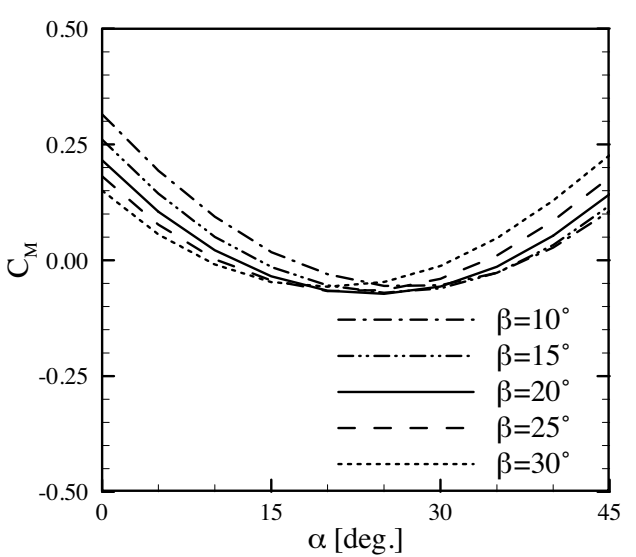

(b) $\gamma=150^{\circ}, V=35^{\circ}, \varphi=5.3^{\circ}$

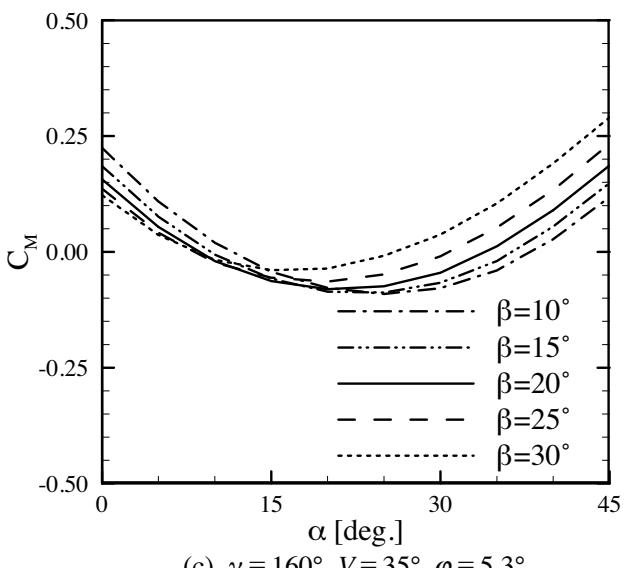

(c) $\gamma=160^{\circ}, V=35^{\circ}, \varphi=5.3^{\circ}$

Fig. 8. Pitching moment coefficient for various flight postures.

stability. ${ }^{31)}$ As shown in Fig. 8 , the values of $\mathrm{d} C_{M} / \mathrm{d} \alpha$ is a negative value for the following flight postures: $\alpha \leq 25^{\circ}$; $\gamma=140^{\circ}, \alpha \leq 23^{\circ}, \gamma=150^{\circ}$; and $\alpha \leq 17^{\circ}, \gamma=160^{\circ}$. This range covers the static stable range in flight. All other ranges are unstable due to $\mathrm{d} C_{M} / \mathrm{d} \alpha>0$.

When the hip angle is increased, the region of the angle of attack is narrowed for stable flight. A small hip angle enhances static stability. The pitching moment coefficient is significantly inclined at a high angle of attack. A large hip angle $(\gamma)$ increases the lift-to-drag ratio. However, the range of stability is constricted.

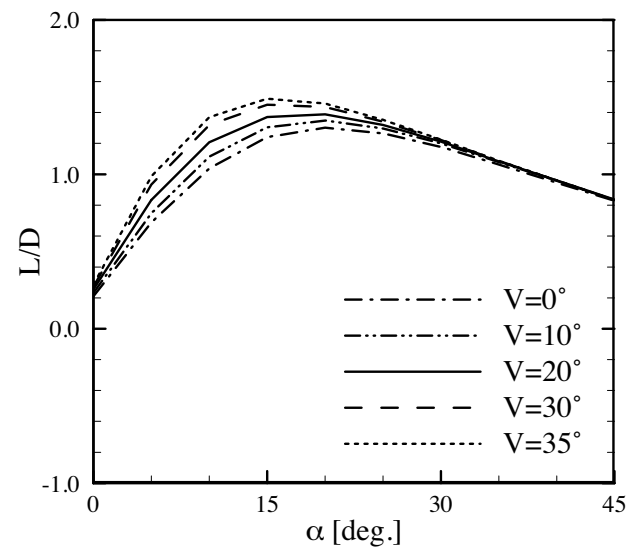

(a) Lift-to-drag ratio

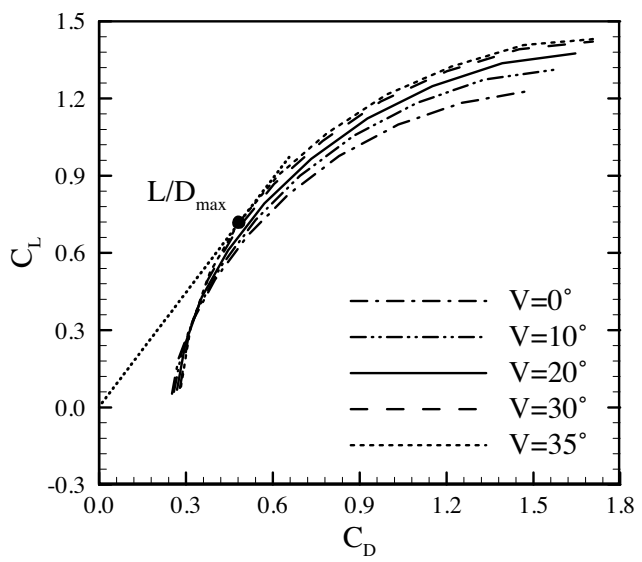

(b) Drag polar

Fig. 9. Aerodynamic characteristics for various ski angles $V \quad\left(\beta=20^{\circ}\right.$, $\left.\gamma=160^{\circ}, \varphi=5.3^{\circ}\right)$.

In other words, the ski jumper should increases the hip angle $(\gamma)$ and overcome an unstable flight posture to improve flight distance.

The ski angle $(V)$ is an effective parameter for ski jumping flight. This parameter is predicted to have a smaller effect on lift-to-drag ratio than $\alpha, \beta$ and $\gamma$ angles due to the small crosssectional area. Figures 9 (a) and (b) show variations of lift-todrag ratio versus angle of attack and the lift coefficients versus the drag coefficients in polar form. The postures of the ski jumper model are set to $\beta=20^{\circ}$ and $\gamma=160^{\circ}$ to evaluate the effect of the ski angle $(V)$.

Depending on the ski angle $(V)$, the maximum lift-to-drag ratio is observed to be at $\alpha=15-20^{\circ}$ in Fig. 9(a). The ski angle augments the lift and drag coefficients, as shown in Fig. 9(b). This augmentation is clearly recognized by comparing the parallel ski angle $\left(V=0^{\circ}\right)$. The lift and drag coefficients particularly depend on ski angle. The largest drag coefficients are achieved at the ski angle of $V=35^{\circ}$. The cause of the increase in aerodynamic coefficient is the enlarged projection area. ${ }^{32)}$ The tendency of the drag polar is changed at $C_{D}<0.32$. In these regions, the angle of attack is less than $10^{\circ}$.

As the ski angle increases, the slope of the drag polar decreases, but the slope of the drag polar increases when the angle of attack $(\alpha)$ is higher than $10^{\circ}$. When the angle of the ski 


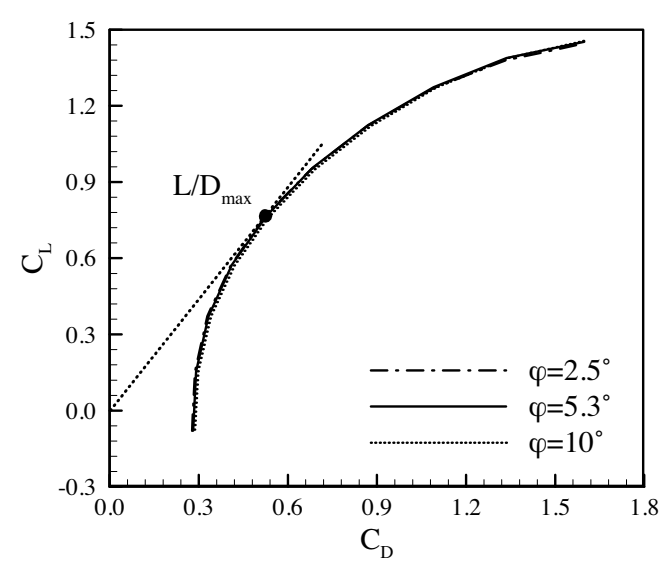

Fig. 10. Lift-to-drag ratio for variation in arm angle $\varphi\left(\beta=9.5^{\circ}\right.$, $\gamma=160^{\circ}, V=35^{\circ}$ ).

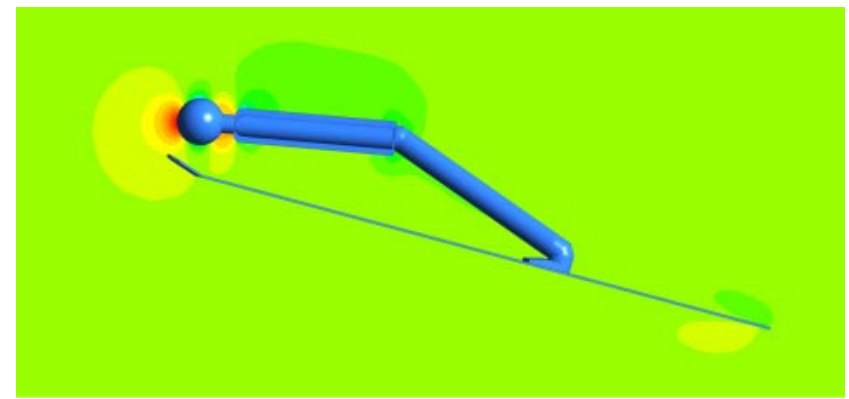

(a) $\alpha=15^{\circ}, \beta=20^{\circ}, \gamma=150^{\circ}, V=35^{\circ}, \varphi=5.3^{\circ}$

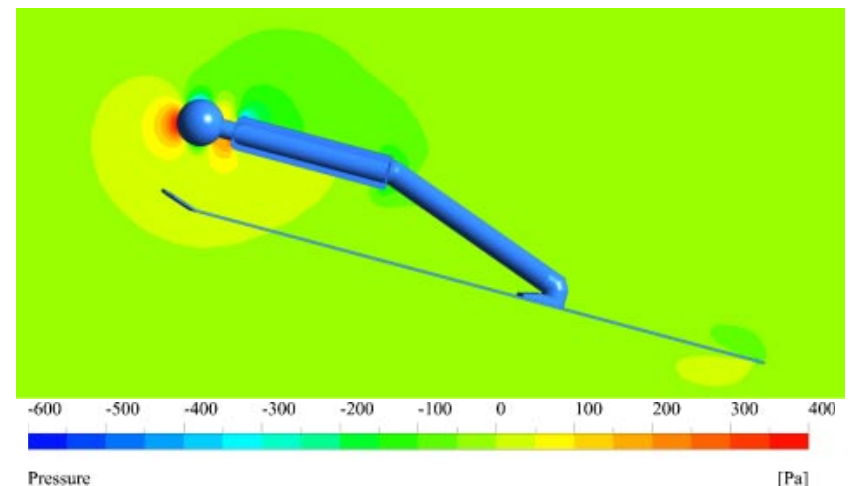

(b) $\alpha=15^{\circ}, \beta=20^{\circ}, \gamma=160^{\circ}, V=35^{\circ}, \varphi=5.3^{\circ}$

Fig. 11. Static pressure contours around the ski jumper model for different hip angles.

$(V)$ increases, lift is greatly increased at a low angle of attack $\left(\alpha<10^{\circ}\right)$. In other words, the lift-to-drag ratio increases significantly when the angle of attack is lower than $10^{\circ}$, as shown in Fig. 9(a). The $L / D_{\max }$ for the ski angle $(V)$ is observed in Fig. 9(b). The $L / D_{\max }$ increases to 1.489 at $V=35^{\circ}$. When a ski jumper attains the optimal V-style flight, the $L / D_{\max }$ is $14.36 \%$ higher than the parallel ski style.

To evaluate the effect of the arm angle $(\varphi)$, the lift and drag coefficients are plotted in Fig. 10. The body angle $(\beta)$ and hip angle $(\gamma)$ are set to $9.5^{\circ}$ and $160^{\circ}$, respectively. In the case of all regions, the effect of the arm angle is remarkably small when changing the arm angle over the range of $\varphi=2.5-10^{\circ}$. With regard to this fact, both the lift and drag coefficients in-

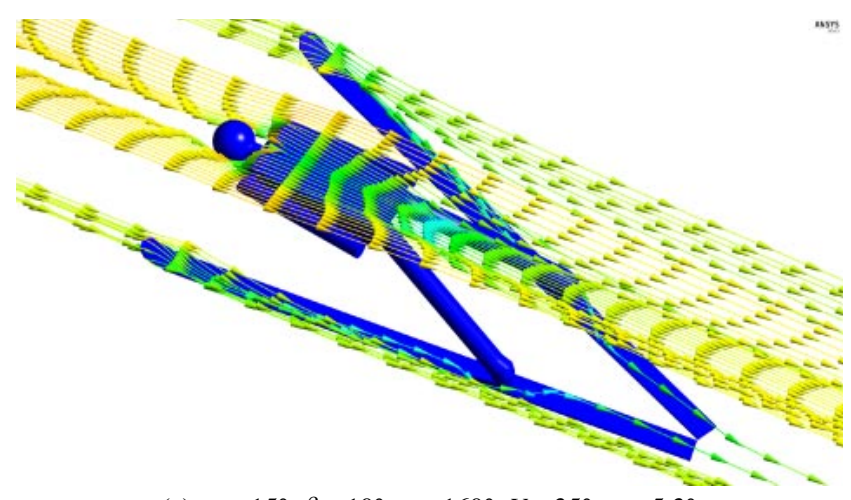

(a) $\alpha=15^{\circ}, \beta=10^{\circ}, \gamma=160^{\circ}, V=35^{\circ}, \varphi=5.3^{\circ}$

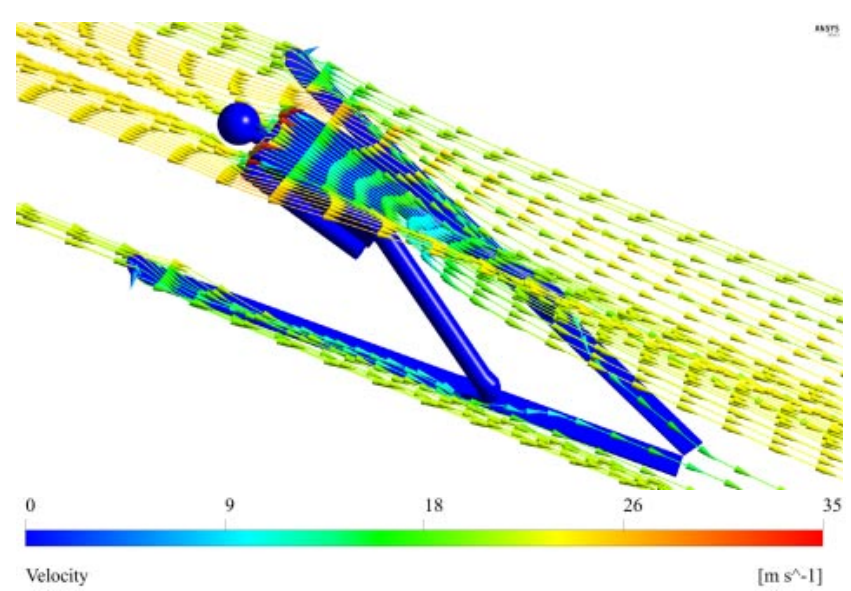

(b) $\alpha=15^{\circ}, \beta=20^{\circ}, \gamma=160^{\circ}, V=35^{\circ}, \varphi=5.3^{\circ}$

Fig. 12. Streamline pattern around the ski jumper model for different body angles relative to the skis.

crease linearly. The $L / D_{\max }$ is 1.465 when the arm angle $(\varphi)$ is $2.5^{\circ}$.

\subsection{Flow field analysis}

The flow fields are analyzed to evaluate the effect of the hip angle $(\gamma)$. Figure 11 shows the contour of the static pressure distributions around the ski jumper model at the angle of attack $\alpha=15^{\circ}$, the body angle relative to the ski plate $\beta=20^{\circ}$ and the hip angle $\gamma=140-160^{\circ}$. The lift force of the ski jumper model is generated by the static pressure difference between the upper surface and the lower surface, similar to the behavior of the airfoil of a wing section. Lower static pressure occurred near the upper part of the ski jumper model. In contrary, high static pressure regions are located beneath the ski jumper. The lift-to-drag ratio of the ski jumper model is increased by increasing of the lower static pressure regions through increasing the hip angle $(\gamma)$.

Figure 12 shows the streamline distributions for the flight posture of the ski jumper model to estimate the effect of body angle $(\beta)$ relative to the skis.

The aerodynamic characteristics for the ski jumper are:

$$
\begin{aligned}
& L / D=1.344, C_{L}=0.539, C_{D}=0.401 \text { at } \beta=10^{\circ}, \\
& L / D=1.457, C_{L}=0.679, C_{D}=0.466 \text { at } \beta=20^{\circ} .
\end{aligned}
$$

Figure 12(b) shows the streamline patterns in the case of a higher lift-to-drag ratio than the case of Fig. 12(a) for the ski jumper model. A fast velocity is observed near the shoulder 


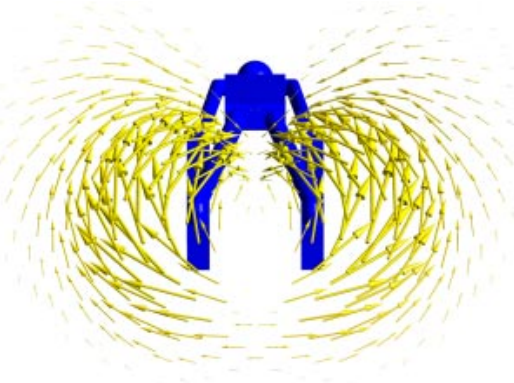

(a) $\alpha=15^{\circ}, \beta=20^{\circ}, \gamma=160^{\circ}, V=0^{\circ}, \varphi=5.3^{\circ}$

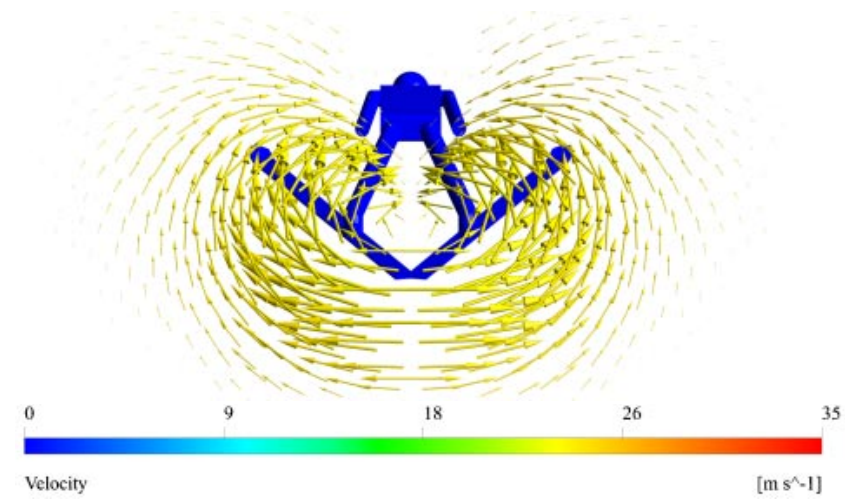

(b) $\alpha=15^{\circ}, \beta=20^{\circ}, \gamma=160^{\circ}, V=35^{\circ}, \varphi=5.3^{\circ}$

Fig. 13. Velocity vector around the ski jumper model for different ski angles.

of the ski jumper. Therefore, the more lift force occurs in the case of $\beta=20^{\circ}$. The downwash, which is produced at the upper surface of the ski jumper, passes through the hip. When the body angle $(\beta)$ is large, more lift and drag forces are created.

In order to compare parallel-style with V-style flight, the velocity vector for the ski jumper model is described in Fig. 13. The cross-sectional views of the velocity vector are plotted behind the ski jumper. The tip vortex from the ski is confirmed in Fig. 13. The lift-to-drag ratio of the Vstyle is higher than that of parallel-style in Fig. 9(a) because the cross-sectional area is broader in V-style flight.

\section{Conclusions}

Computational analysis was performed for various ski jumper postures during flight. A simulation of the flow field around the ski jumper was performed based on RANS equations using commercial software.

In the present study, we concentrated on a better understanding of the aerodynamic characteristics of ski jumping during flight. The range of ski jumping is set to be as wide as possible and to include real positions. The range of hip angle $(\gamma)$, which was determined based on that of a world-class ski jumper, was calculated and set from $140^{\circ}$ to $160^{\circ}$. The results of the simulated lift-to-drag ratios were in good agreement with the experimental results. ${ }^{11)}$

The hip and body angles for the ski jumper model are more effective in flight. The lift-to-drag ratio of the ski jumper model is directly proportional to the hip angle. However, when the hip angle is increased, the region of the angle of attack for stable flight is narrowed. A small hip angle enhances static stability. The pitching moment coefficient is considerably inclined in the region of high angle of attack. The body angle is likely to resemble that of camber in an airfoil; therefore, changing the body angle can improve the useful angle of attack.

Velocity is affected by the shoulders of the ski jumper. The downwash, which is caused from the upper surface of the body, passes around the hip.

The ski angle gradually augments the lift-to-drag ratio. This augmentation is clearly appreciated when compared to the case of a parallel ski angle. The effect of the arm angle is markedly small.

In conclusion, ski jumpers should increase the hip angle $(\gamma)$ during flight to enhance flight distance and overcome unstable flight. The effect of crosswind, dynamic changes in the wind and fabric roughness are not considered in this study. These effects should be considered in future studies. Environmental conditions could also be included.

\section{Acknowledgments}

This research was supported by the Basic Science Research Program of the National Research Foundation of Korea (NRF), funded by the Ministry of Education (No. 2009-009072) for Jinsoo Cho. This research was also financially supported by Hansung University for Leesang Cho.

\section{References}

1) David, L. and Karen, C.: Encyclopedia of World Sport, Oxford University Press, New York, 1999, pp. 357-358.

2) Straumann, R.: Vom Skiweitsprung und seiner Mechanik (1 Teil) [Ski Jumping and the Mechanics Theory], Jahrbuch des Schweizerischen Skiverbandes, 22 (1926), pp. 11-29.

3) Hochmuth, G.: Untersuchungen über den Einfluß der Absprungbewegung auf die Sprungweite beim Skispringen, Wissenschaftliche Zeitschrift der Deutschen Hochschule für Körperkultur, 1 (1958/59), pp. 29-57.

4) Müller, E. and Schwameder, H.: Biomechanical Aspects of New Techniques in Alpine Skiing and Ski-jumping, J. Sports Sci., 21 (2003), pp. 679-692.

5) Schwameder, H. and Müller, E.: Biomechanics in Ski Jumping: A Review, Eur. J. Sport Sci., 1 (2001), pp. 1-16.

6) Schwameder, H.: Biomechanics Research in Ski Jumping, 1991-2006, Sports Biomechanics, 7 (2008), pp. 114-136.

7) Remizov, L. P.: Biomechanics of Optimal Flight Ski-jumping, J. Biomechanics, 17 (1984), pp. 167-171.

8) Denoth, J., Luethi, S. M., and Gasser, H. H.: Methodological Problems in Optimization of the Flight Phase in Ski Jumping, J. Biomechanics, 3 (1987), pp. 404-418.

9) Müller, W., Platzer, D., and Schmölzer, B.: Dynamics of Human Flight on Skis: Improvements in Safety and Fairness in Ski Jumping, J. Biomechanics, 29 (1996), pp. 1061-1068.

10) Schmölzer, B. and Müller, W.: The Importance of Being Light: Aerodynamic Forces and Weight in Ski Jumping, J. Biomechanics, 35 (2002), pp. 1059-1069.

11) Schmölzer, B. and Müller, W.: Individual Flight Styles in Ski Jumping: Results Obtained during Olympic Games Competitions, J. Biomechanics, 38 (2005), pp. 1055-1065.

12) Meile, W., Reisenberger, E., Mayer, M., Schmölzer, B., Müller, W., and Brenn, G.: Aerodynamics of Ski Jumping: Experiments and CFD Simulations, Exp. Fluids, 41 (2006), pp. 949-964. 
13) Jin, H., Shimizu, S., Watanuki, T., Kubota, H., and Kobayashi, K. Desirable Gliding Styles and Techniques in Ski Jumping, J. Appl. Biomechanics, 11 (1995), pp. 460-474.

14) Watanabe, K. and Watanabe, I.: Aerodynamics of Ski Jumping: Effect of "V-style" to Distance. XIVth Congress of the International Society of Biomechanics, 1993, pp. 1452-1453.

15) Seo, K., Watanabe, I., and Murakami, M.: Aerodynamic Force Data for a V-Style Ski Jumping Flight, ISEA Sports Engineering, 7 (2004), pp. 31-39.

16) Seo, K., Murakami, M., and Yoshida, K.: Optimal Flight Technique for V-style Ski Jumping, ISEA Sports Engineering, 7 (2004), pp. 97-104.

17) Marqué-Bruna, P. and Grimshaw, P.: Mechanics of Flight in Ski Jumping: Aerodynamic Stability in Pitch, Sports Technol., 2 (2009), pp. 24-31.

18) Fédération Internationale de Ski: The International Ski Competition Rules Book III Ski Jumping, Oberhofen, Switzerland, 2013.

19) ANSYS CFX-14: 5 Solver Theory, Ansys Inc., 2012.

20) Menter, F. R.: Two-equation Eddy Viscosity Turbulence Models for Engineering Applications, AIAA J., 32 (1994), pp. 1598-1605.

21) Park, S. H. and Kwon, J. H.: Implementation of k-w Turbulence Models in an Implicit Multigrid Method, AIAA J., 42 (2004), pp. 1348-1357.

22) Guilmineau, E., Piquet, J., and Queutey, P.: Two-Dimensional Turbulent Viscous Flow Simulation Past Airfoils at Fixed Incidence, Comput. Fluids, 26 (1997), pp. 135-162.
23) Murayama, M., Lei, Z., Mukai, J., and Yamamoto, K.: CFD Validation for High-Lift Devices: Three-Element Airfoil, T. Jpn. Soc. Aeronaut. Space Sci., 49 (2006), pp. 40-48.

24) Rodi, W. and Scheurer, G.: Scrutinizing the k- $\varepsilon$ : Model under Adverse Pressure Gradient Conditions, J. Fluid Eng., 108 (1986), pp. 174-179.

25) Menter, F. R.: Influence of Freestream Values on $\kappa$ - $\omega$ Turbulence Model Predictions, AIAA J., 30 (1992), pp. 1651-1659.

26) Johnson, D. A. and King, L. S.: Mathematically Simple Turbulence Closure Model for Attached and Separated Turbulent Boundary Layers, AIAA J., 23 (1985), pp. 1684-1692.

27) Wilcox, D. C.: Turbulence Modeling for CFD, 2nd Ed., DCW Industries, California, 1998, pp. 13-18.

28) Reisenberger, E., Meile, W., Brenn, G., and Müller, W.: Aerodynamic Behaviour of Prismatic Bodies with Sharp and Rounded Edges, Exp. Fluids, 37 (2004), pp. 547-558.

29) Meile, W., Müller, W., and Reisenberger, E.: Ski-Jumping Aerodynamics: Model-Experiments and CFD-Simulations. Sport Aerodynamics, Springer, Vienna, 2008, pp. 183-216.

30) McCormick, B. W.: Aerodynamics, Aeronautics, and Flight Mechanics, Wiley, New York, 1995, pp. 69-72.

31) John, D. A., Jr.: Introduction to Flight, McGraw-Hill, New York, 2005, pp. 527-531.

32) Ito, S., Seo, K., and Asai, T.: An Experimental Study on Ski Jumping Styles (P140), The Engineering of Sport 7, Springer, Paris, 2008, pp. 9-17. 